\title{
Identification of chromoblastomycosis agents by Fourier Transform-Infrared Spectroscopy supervised by ITS rDNA region
}

\author{
Daiane Heidrich 1,2; Valeriano Antonio Corbellini 3; Mauricio Ramírez-Castrillón 4; Carina
}

Timotheo 5; Danielle Machado Pagani 5; Maria Lúcia Scroferneker 1,2

1 Postgraduate Program in Medicine: Medical Sciences, Universidade Federal do Rio Grande do Sul, Brazil.

2 Department of Microbiology, Immunology and Parasitology, ICBS, Universidade Federal do Rio Grande do Sul, Brazil.

3 Department of Chemistry and Physics, Postgraduate Program in Health Promotion, Postgraduate Program in

Environmental Technology, Universidade de Santa Cruz do Sul, Brazil.

4 Research group in mycology (GIM/CICBA), Faculty of Basic Sciences, Universidad Santiago de Cali, Colombia.

5 Postgraduate Program in Agricultural and Environmental Microbiology, Universidade Federal do Rio Grande do Sul,

Brazil.

Objectives: differentiate the chromoblastomycosis (CBM) agents for identification at level species using Fourier Transform-Infrared Spectroscopy (FTIR) supervised by sequencing of Internal Transcribed Spacer (ITS) rDNA region.

Methods: seventy-seven isolates of five genera of CBM agents were identified by comparison of ITS sequences of type strains available in GenBank using BLAST algorithm. Phylogenetic trees were performed when necessary for differentiation of Fonsecaea pedrosoi and Fonsecaea monophora. The strains prepared for Attenuated Total Reflection (ATR) with a new methodology using slices in glass fixing-modeling proposed. Five spectra were recorded from 4000 to $650 \mathrm{~cm}^{-1}$ for a strain and the average spectra of each one was calculated. The data set was pre-processed by sample (normalization and multiplicative scatter correction-MSC) and by variable (meancentering) in Pirouette ${ }^{\circledR}$ Software. The algorithms used for analyzing the spectra were Hierarchical cluster analysis (HCA) to observe similarities and differences between species and Partial Least Square Regression (PLS) to propose the model of identification, which was evaluated by cross-validation and external validation.

Results: the strains were identified with $99 \%$ of identity with type strain of each species of thirteen species distributed on five genera of CBM. The identified species were: Fonsecaea pedrosoi (41), Fonsecaea monophora (15), Fonsecaea pugnacious (1), Fonsecaea nubica (1), Cladophialophora carrionii (3), Cladophialophora bantiana (1), Phialophora verrucosa (2), Phialophora americana (5), Exophiala spinifera (3), Exophiala xenobiotica (2), Rhinocladiella quaspersa (1), Rhinocladiella tropicalis (1) and Rhinocladiella similis (1). Dendrogram of HCA had similar result to phylogenic tree when comparing F. pedrosoi and F. monophora, since these species presented $81.2 \%$ and $78 \%$ of similarity by the two tools, respectively. In relation to regions bands of FTIR obtained, the regions between 4000 and $3100 \mathrm{~cm}^{-1}$ (attributed to $\mathrm{NH}$ and $\mathrm{OH}$ stretching) together with the bands 1650 (amide I of proteins), 1050 and 1025 (polysaccharides bands) and the 900-800 region (fingerprint) (Figure 1). It was the fraction of spectra of FTIR most related to the differences between CBM species, as observed by regression vector in PLS. The model proposed could classify the thirteen CBM species, with high coefficient of determination and low error (RMSECV) of the tendency line obtained (Figure 2), indicating that this model can be efficient to identify these species of CBM agents.

Conclusion: CBM is a fungal infection caused by species of different dematiaceous genera that are identified by sequencing of one or more regions of DNA and phylogenic tree, which become an expensive and difficult work. FTIR demonstrated to be a faster and inexpensive alternative for identification of CBM agents.

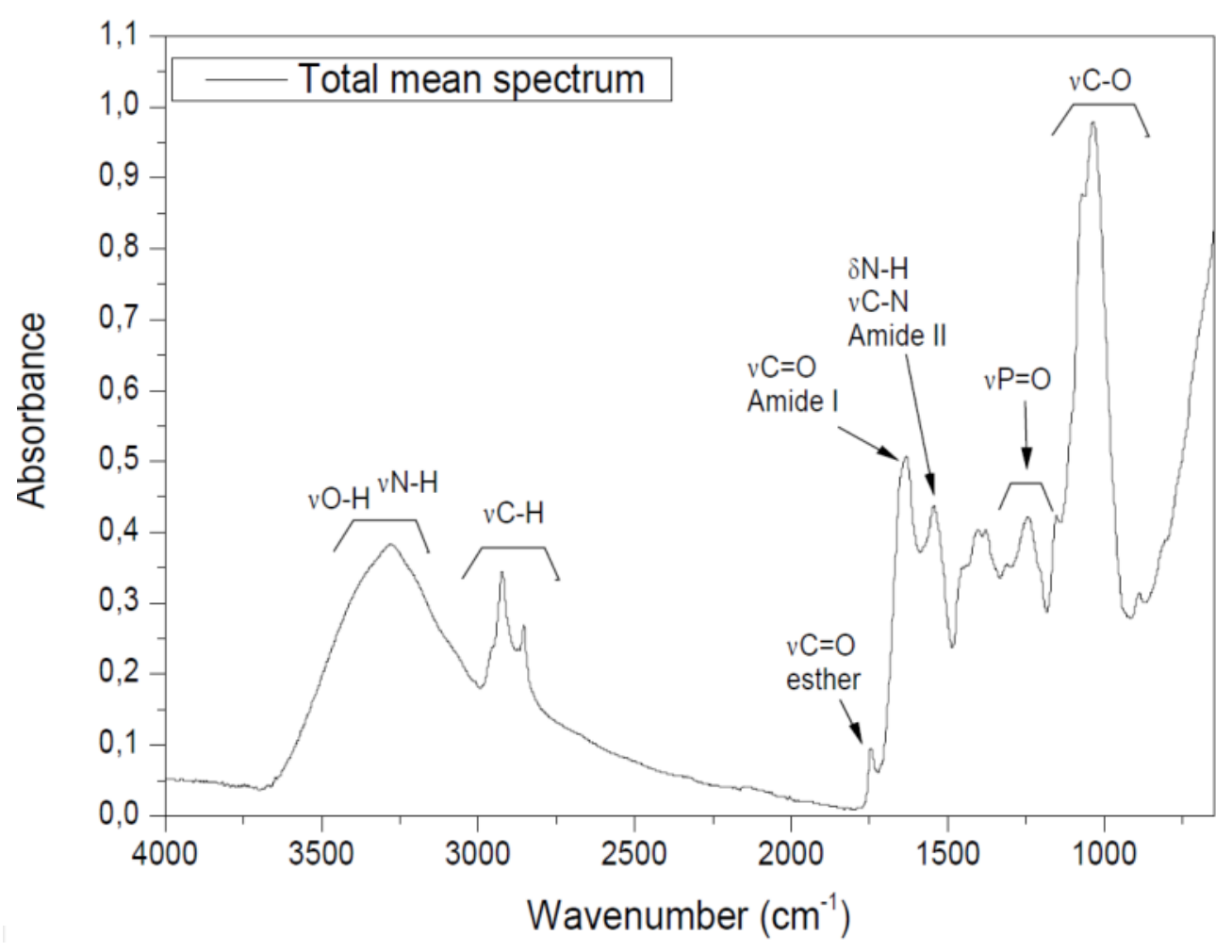

Figure 1. FTIR total spectrum presenting the bands found in chromoblastomycosis agents

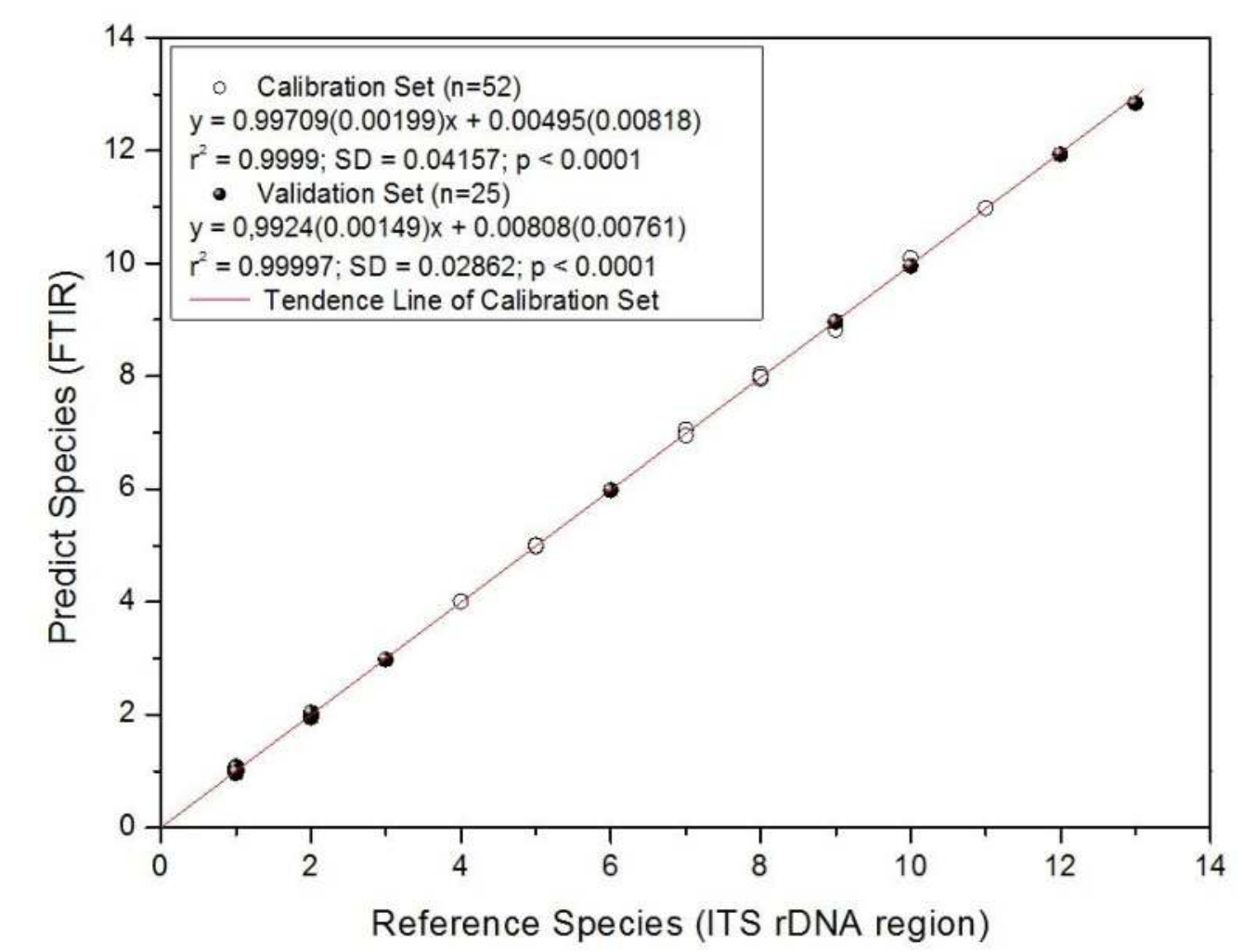

Figure 2. Calibration set and validation set curves of the chromoblastomycosis agents species identified by DNA analysis of ITS region with the species predicted by the PLSR model. The codes refers to: 1. F. pedrosoi, 2. F. monophora, 3. F. nubica, 4. F. pugnacious, 5. C. carrionii, 6. C. bantiana, 7. P. verrucosa, 8. P. americana, 9. E. spinifera, 10. E. xenobiotica, 11. R. tropicalis, 12. $R$. aquaspersa, 13. R. similis. 